\title{
Long-term nitrofurantoin: an analysis of complication awareness, monitoring, and pulmonary injury cases
}

\begin{abstract}
Toby Peter Speirs ${ }^{1}$, Nicole Tuffin 1 , Finlay Mundy-Baird', Helena Sakota', Sarah Mulholland', Michelle Westlake ${ }^{1}$, Max Lyon ${ }^{1}$, Andrew R Medford ${ }^{1}$, Charles Sharp ${ }^{1}$, Michael Darby², Mahableshwar Albur ${ }^{3}$, Francis Keeley4, Helena Burden ${ }^{4}$, Charlie Kenward ${ }^{5}$, Elizabeth Jonas ${ }^{5}$, Shaney Barratt ${ }^{1}$, Huzaifa I Adamali ${ }^{1 *}$
\end{abstract}

${ }^{1}$ Bristol Interstitial Lung Disease Service, Southmead Hospital, North Bristol NHS Trust, Bristol, UK; ${ }^{2}$ Department of Radiology, North Bristol NHS Trust, Bristol, UK; ${ }^{3}$ Department of Microbiology and Infectious Disease, North Bristol NHS Trust, Bristol, UK; ${ }^{4}$ Department of Urology, North Bristol NHS Trust, Bristol, UK; ${ }^{5} \mathrm{NHS}$ Bristol, North Somerset and South Gloucestershire Clinical Commissioning Group, Bristol, United Kingdom

\begin{abstract}
Background: Long-term nitrofurantoin (NF) treatment can result in pulmonary and hepatic injury. Current guidelines do not outline the type or frequency of monitoring required for detection of these injuries.
\end{abstract}

Aim: To assess 1) awareness of NF complications among prescribers; 2) monitoring practice; and 3) to describe the pulmonary sequelae of NF-related complications.

Design \& setting: Evaluation of prescribing habits by questionnaires and review of GP databases, and case-note review in secondary care.

Method: The following study procedures were undertaken: 1) an electronic questionnaire was distributed to prescribers, interrogating prescribing and monitoring practices, and awareness of complications; 2) an analysis was undertaken (June-July 2020) of NF monitoring among GPs in the local clinical commissioning group (CCG); and 3) a case review was carried out of patients diagnosed with NF-induced interstitial lung disease (NFILD) at the interstitial lung disease (ILD) centre (2014-2020).

Results: A total of 125 prescribers of long-term NF responded to the questionnaire $(82.4 \%$ GPs; $12.0 \%$ urologists). Many were unaware of the potential for liver (42.4\%) and lung (28.0\%) complications; $40.8 \%$ and $52.8 \%$ never monitored for these, respectively. Only $53.3 \%$ of urologists believed themselves responsible for arranging monitoring, while nearly all GPs believed this to be the prescriber's responsibility (94.2\%). One-third of all responders considered current British National Formulary (BNF) guidelines 'not at all sufficient/clear', with mean clarity scoring of 2.2/5. Among patients with NFILD $(n=46)$, NF had been prescribed most often (69.6\%) for treatment of recurrent UTI and $58.6 \%$ ( $n$ $=27$ ) were prescribed for $>6$ months. On withdrawal of the medication $61.4 \%$ displayed resolution (completely or minimal fibrosis), while $15.9 \%$ of patients had progressive lung fibrosis.

Conclusion: NF can cause marked or irreversible lung complications and there is currently a shortfall in awareness and monitoring. Existing monitoring guidelines should be augmented.

\section{How this fits in}

It is known that long-term NF use can induce hepatic and/or pulmonary complications, and that monitoring is required for early detection. This research highlights a shortfall in 1) awareness of NF- 
related side effects and 2) monitoring practice in the prescribing community. Hence, the following points are suggested: awareness of potential NF complications should be raised among GPs and urologists; monitoring responsibilities need to be clarified; and existing monitoring guidelines should be augmented.

\section{Introduction}

NF is prescribed for treatment of acute urinary tract infections (UTIs) and prophylactically to reduce UTI recurrence. ${ }^{1,2}$ Complications, notably hepatic and pulmonary, have been associated with short-term and long-term use and are more common in women. ${ }^{3-5}$ NFILD refers to a spectrum of lung changes, from an acute hypersensitivity reaction (within 1-2 weeks) to a chronic pulmonary reaction involving fibrosis (months to years of exposure). The prevalence of severe adverse events was estimated at $0.2 \%(95 \%$ confidence interval $[\mathrm{Cl}]=<0.01 \%$ to $1.2 \%)$ in controlled trials and from 0.02 to 1.5 events per $1000 \mathrm{NF}$ users in observational studies. ${ }^{6}$ Although severe adverse events may be uncommon in a general population, the risk increases with longer treatment durations and the reported prevalence among individuals aged $\geq 65$ years is $2.1 \% .^{5}$ The precise mechanisms of adverse effects are not fully understood. ${ }^{7-9}$

Current adult BNF guidelines state: 'On long-term therapy, monitor liver function and monitor for pulmonary symptoms, especially in the elderly (discontinue if deterioration in lung function). ${ }^{10}$ However, neither a method nor frequency of hepatic or pulmonary monitoring, nor a definition for 'long term' is suggested. National Institute for Health and Care Excellence (NICE) guidance on antimicrobial prescribing for recurrent UTI recommend review at 6 months. ${ }^{11}$ Both NICE guidance and local CCG guidelines rely on referencing BNF advice. ${ }^{2}$

Negligence in NF monitoring has been highlighted as a significant cause of litigation, according to the UK Medical Protection Society (UKMPS) ${ }^{12}$ that advises liver function testing (LFT) and reviews for respiratory symptoms at least 6-monthly, with consideration of more frequent monitoring. ${ }^{12}$

Improving monitoring protocols may speed up recognition of complications and withdrawal of NF to lessen severity of toxicity. The current landscape of monitoring practice is unknown. This study aimed to determine: 1) the awareness of NF side effects; 2) the existing monitoring practices of NF in general practice within the local area; and 3) to describe the cohort of patients diagnosed with NFILD by multidisciplinary team (MDT) consensus at the Bristol Interstitial Lung Disease (BILD) service.

\section{Method}

\section{Setting}

This study involved collaboration of the BILD service at North Bristol NHS Trust (NBT) and the Bristol, North Somerset and South Gloucestershire (BNSSG) CCG. The BILD service provides secondary and tertiary specialist ILD care to a catchment of 1.2 million in South West England.

\section{Study procedures}

\section{Questionnaire: assessing prescribing insight}

A 13-item questionnaire was electronically distributed (July 2020) to all local GPs ( $n=675)$, nurses ( $n=145)$, CCG medicine optimisation pharmacists (MOPs; $n=72)$, and South West urologists $(n=$ 130) (see Supplementary Appendix S1). Responses were anonymised to ensure confidentiality and encourage candid responses.

\section{Analysis of monitoring practices of NF within primary care}

Data were requested from all CCG GP practices $(n=78)$, collated by MOPs, and submitted to study organisers for analysis. Monitoring history was analysed for all patients with an active NF prescription at the time of data collection (26 June-25 July 2020) and registered at a participating GP practice. Patient demographics and extent of NF exposure (dose and duration: episodic, continuous, or cyclical use) were collected from GP practice records. Data regarding relevant monitoring procedures performed at baseline and subsequently were also collated, including clinical examination, oxygen 
saturation, chest X-ray (CXR), spirometry, and LFTs. Only patients with a NF prescription initiated within the preceding 2 years were included, to address contemporary practice.

\section{Case-note review and characterisation of patients with an MDT consensus diagnosis of NFILD}

Patients with an MDT consensus diagnosis of NFILD (September 2015-July 2020) were retrospectively identified from the BILD database. From records of patients diagnosed with NFILD, data were collated regarding basic demographics and NF exposure. Baseline (initial presentation) and final (completion of audit) lung function were documented. Spirometry measurements included forced vital capacity $(F V C)$, forced expiratory volume in 1 second (FEV1), diffusion capacity of the lungs (TLCO), and TLCO with correction for haemoglobin (KCO). Six-minute walking distance (6MWT), oxygen saturations, and MRC dyspnoea scores were also compared between initial and final visits. Baseline and final high-resolution computerised tomography (HRCT) imaging results were independently reviewed for changes to radiological pattern by a thoracic radiologist and a respiratory consultant. Treatment regimens including long-term or ambulatory oxygen therapy (LTOT or AOT) and steroids were interrogated.

\section{Statistical analysis}

Continuous variables were tested for normality using the D'Agostino-Pearson test. Where parametric, data were tested by paired two-tailed t-tests and presented as mean \pm standard deviation (SD). Where non-parametric, data were tested by Wilcoxon tests (paired) or Mann-Whitney $U$ tests (unpaired) and presented as medians with interquartile range (IQR). For all statistical tests, $P \leq 0.05$ was considered statistically significant. Data were analysed using GraphPad Prism 8 for Windows (version 8.4.3).

\section{Results}

\section{Questionnaire: assessing prescribing insight}

Response rate among GPs and urologists, respectively, was 35.9\% $(n=242)$ and 31.5\% $(n=41)$. Longterm (>6 months) NF was prescribed by $39.8 \%(n=125)$ of the 314 total responders: GPs $82.4 \%(n=$ 103), urologists $12.0 \%(n=15)$.

CCG guidelines were preferred $(47.2 \%, n=59)$, followed by NICE $(38.4 \%, n=48)$, and local trust guidelines $(10.4 \%, n=13)$. The questionnaire also found $11.2 \%(n=14)$ did not use guidelines and $65.6 \%$ $(n=82)$ of prescriptions were for recurrent UTIs.

For treatment of recurrent UTIs, most prescribers based their antibiotic choice on culture results $(52.8 \%, n=66)$. However, the first-choice treatment was NF for $28.0 \%(n=35)$ and trimethoprim for $18.4 \%(n=23)$.

Many prescribers were unaware of the potential hepatotoxicity $(42.4 \%, n=53)$ or pulmonary toxicity $(28.0 \%, n=35)$. In addition, $52.8 \%(n=66)$ of prescribers reported that they did not measure baseline function at prescription (respiratory symptoms, oxygen saturation, respiratory examination,

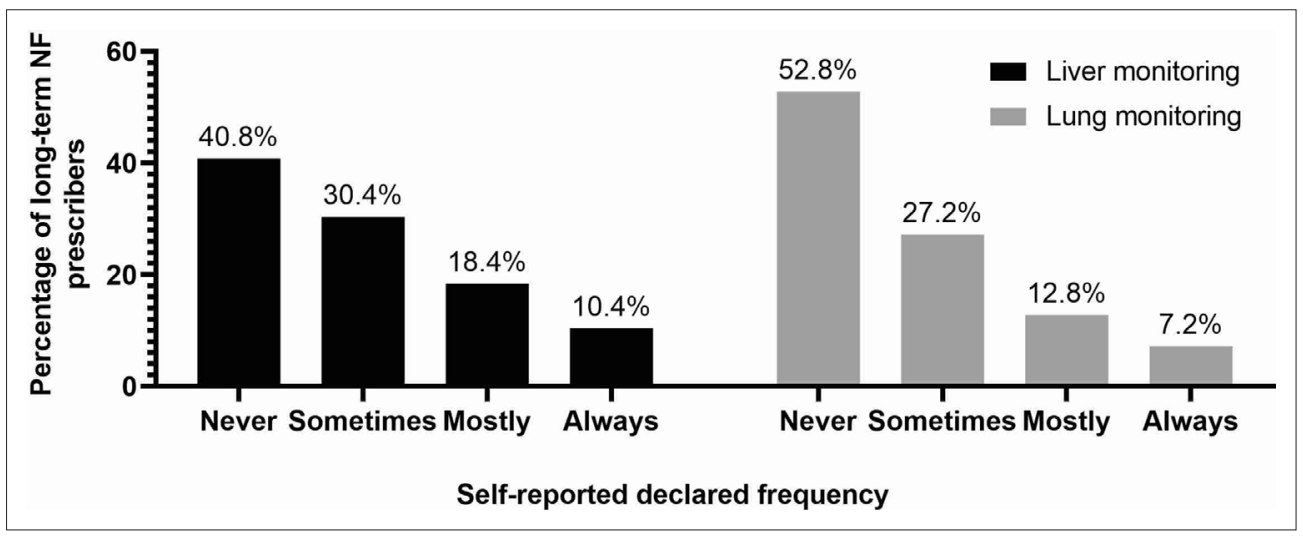

Figure 1 Frequency with which long-term prescribers of NF self-reported initiating a monitoring programme for patients using NF. NF = nitrofurantoin. 


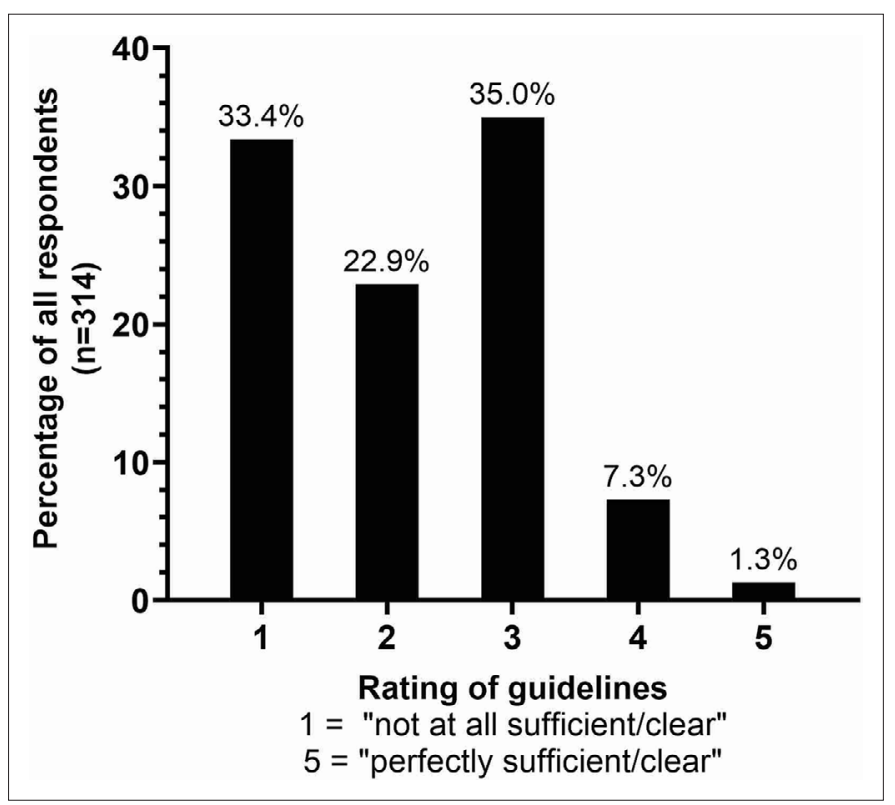

Figure 2 Reported quality rating of current prescription guidelines for NF, across all responders of the questionnaire. NF = nitrofurantoin.

CXR, spirometry, and LFTs). The most common baseline test or examination was LFT $(32.8 \%, n=41)$, while $20.8 \%(n=26)$ documented baseline respiratory symptoms. A minority $(<5 \%)$ checked other modalities (oxygen saturations, respiratory examination, CXR, and spirometry) and some mentioned taking a history of previous lung and/or liver abnormalities and blood tests to assess for infection.

The questionnaire investigated frequency of follow-up monitoring. LFT was reportedly 'always' monitored by $10.4 \%(n=13)$ and 'lung-related examination and tests (LuRT)' 'always' by $7.2 \%$ ( $n=$ 9) of prescribers. The most common tendency was to 'never' monitor liver $(40.8 \%, n=51)$ or lung function $(52.8 \%, n=66)$ (Figure 1). When prescribers did monitor, this was most commonly performed every 12 months $(32.0 \%, n=40)$.

The questionnaire gauged agreement with this statement: 'The prescriber of nitrofurantoin is responsible for monitoring the drug side effects/complications. 'Nearly all GPs $(94.2 \%, n=97)$ considered monitoring to be the responsibility of the prescriber in contrast to $53.3 \%(n=8)$ of urologists.

The questionnaire interrogated the perceived clarity and sufficiency of current guidelines for long-term NF prescription. One-third of responders $(n=105)$ considered guidelines to be 'not at all sufficient/clear' whereas $1.3 \%(n=4)$ considered them to be 'perfectly sufficient/clear' (Figure 2$)$. The mean rating of existing guidelines was 2.2/5 (SD 1.0).

\section{Analysis of monitoring practices of NF within primary care}

Data were collated from 62 of 78 GP practices in the CCG (79.5\%). A total of 503 patients used long-term NF (July 2020). Those with NF prescription initiated within the preceding 2 years are considered herein $(n=265)$. The cohort was predominantly female $(81.5 \%, n=216)$ and aged $>60$ years (Table 1 )

Baseline monitoring (tests undertaken at prescription date \pm 1 month) of any kind was absent in $73.6 \%(n=195)$ of patients; $11.7 \%$ ( $n$ $=31)$ received only LFTs and $10.2 \%(n=27)$ only LuRT at baseline. The remaining minority $(4.5 \%, n$ $=12$ ) had both LFT and LuRT at baseline.

A subcohort of patients $(39.6 \%, n=105)$ had been on NF for 6-24 months. Of these,
Table 1 Age distribution of the 503 patients prescribed long-term (>6 months) NF at GP practices $(n=78)$ within the BNSSG CCG.

\begin{tabular}{lc}
\hline Age range, years & $\%$ \\
\hline $0-20$ & 3.0 \\
\hline $21-40$ & 18.1 \\
\hline $41-60$ & 25.3 \\
\hline $61-80$ & 38.1 \\
\hline 881 & 15.5 \\
\hline
\end{tabular}

BNSSG CCG $=$ Bristol, North Somerset and South Gloucestershire clinical commissioning group. NF = nitrofurantoin. 
Table 2 Baseline characteristics of BILD patients with NF-induced interstitial lung disease (NFILD).

\begin{tabular}{lc}
\hline Characteristic & Mean $\pm \mathrm{SD}^{\mathrm{a}}$ \\
\hline Male:female ratio & $9: 37$ \\
\hline Age, years & $71.76 \pm 1.62$ \\
\hline Lung Function & \\
\hline FVC (L) & $2.30 \pm 0.11$ \\
\hline FEV1 (L) & $1.82 \pm 0.07$ \\
\hline Ratio (\%) & $79.8 \pm 1.43$ \\
\hline TLCO (mmol/min/kPa) & $4.10 \pm 0.23$ \\
\hline KCO (mmol/(min/kPa/L) & $1.27 \pm 0.08$ \\
\hline Six-minute walk test & \\
\hline Distance walked (m) & $270.3 \pm 22.8$ \\
\hline Minimum desaturation (\%) & $89.5 \pm 0.87$ \\
\hline MRC dyspnoea score & $3.3 \pm 0.17$ \\
\hline
\end{tabular}

anless otherwise stated. BILD = Bristol Interstitial Lung Disease. FEV1 = forced expiratory volume in 1 second. FVC = forced vital capacity. $\mathrm{KCO}=\mathrm{TLCO}$ correction for haemoglobin. MRC = Medical Research Council. TLCO = diffusion capacity of the lungs.

cystitis.

The duration of NF exposure preceding toxicity ranged from $<1$ week (acute adverse effect) to $\geq 12$ months. Exposure duration was 6-12 months for $19.6 \%(n=9)$ and $\geq 12$ months for $39.1 \%(n=18)$. Duration was unknown for $32.6 \%(n=15)$ of patients.

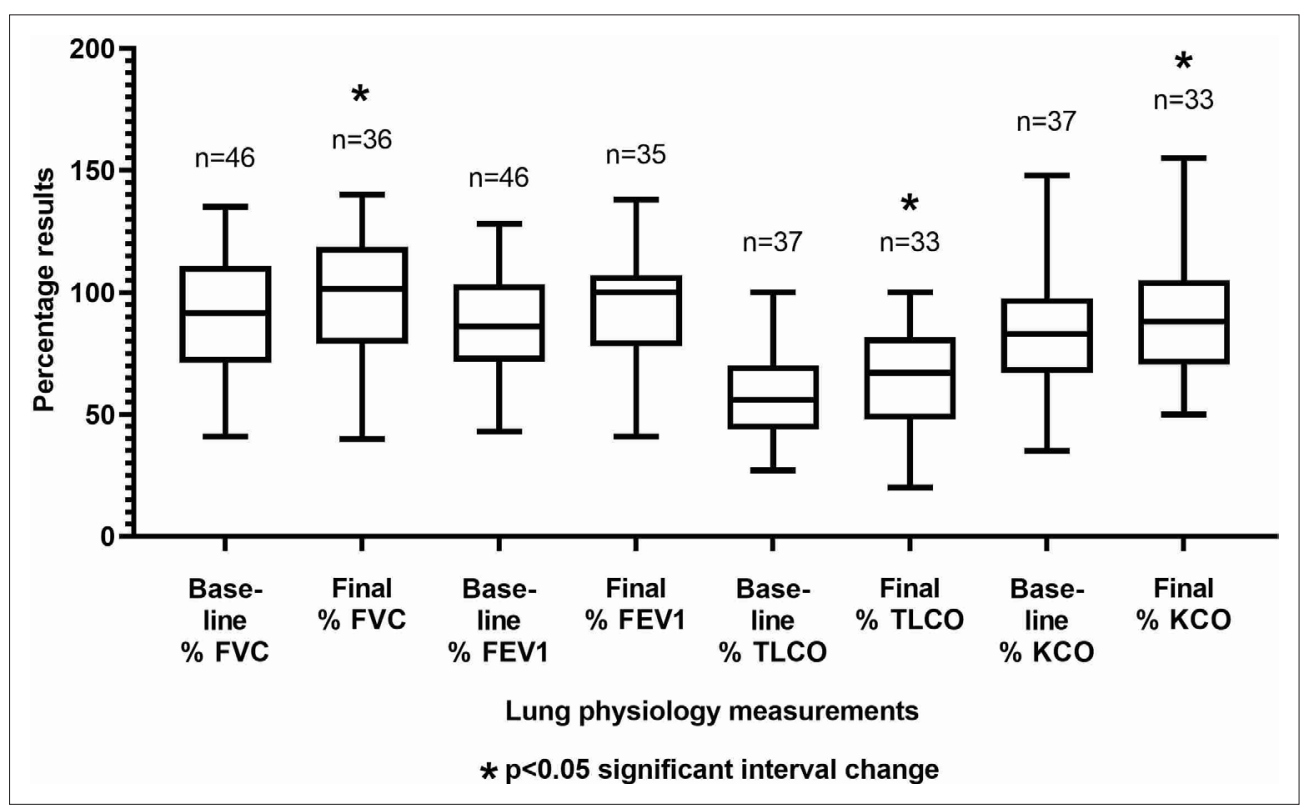

Figure 3 Spirometry test result changes over interval of Bristol Interstitial Lung Disease clinic treatment for nitrofurantoin-induced interstitial lung disease. FVC increased by median $5.00 \%(95 \% \mathrm{Cl}=-0.79$ to 15.18$)$. FEV1 increased by mean $2.93 \%(95 \% \mathrm{Cl}=-4.86$ to 10.72$)$. TLCO increased by mean $7.70 \%(95 \% \mathrm{Cl}=2.49$ to 12.91$)$. $\mathrm{KCO}$ increased by mean $7.10 \%(95 \% \mathrm{Cl}=2.27$ to 11.94$)$. $\mathrm{FEV} 1=$ forced expiratory volume in 1 second. $\mathrm{FVC}=$ forced vital capacity. $\mathrm{KCO}=$ TLCO correction for haemoglobin. TLCO = diffusion capacity of the lungs. 


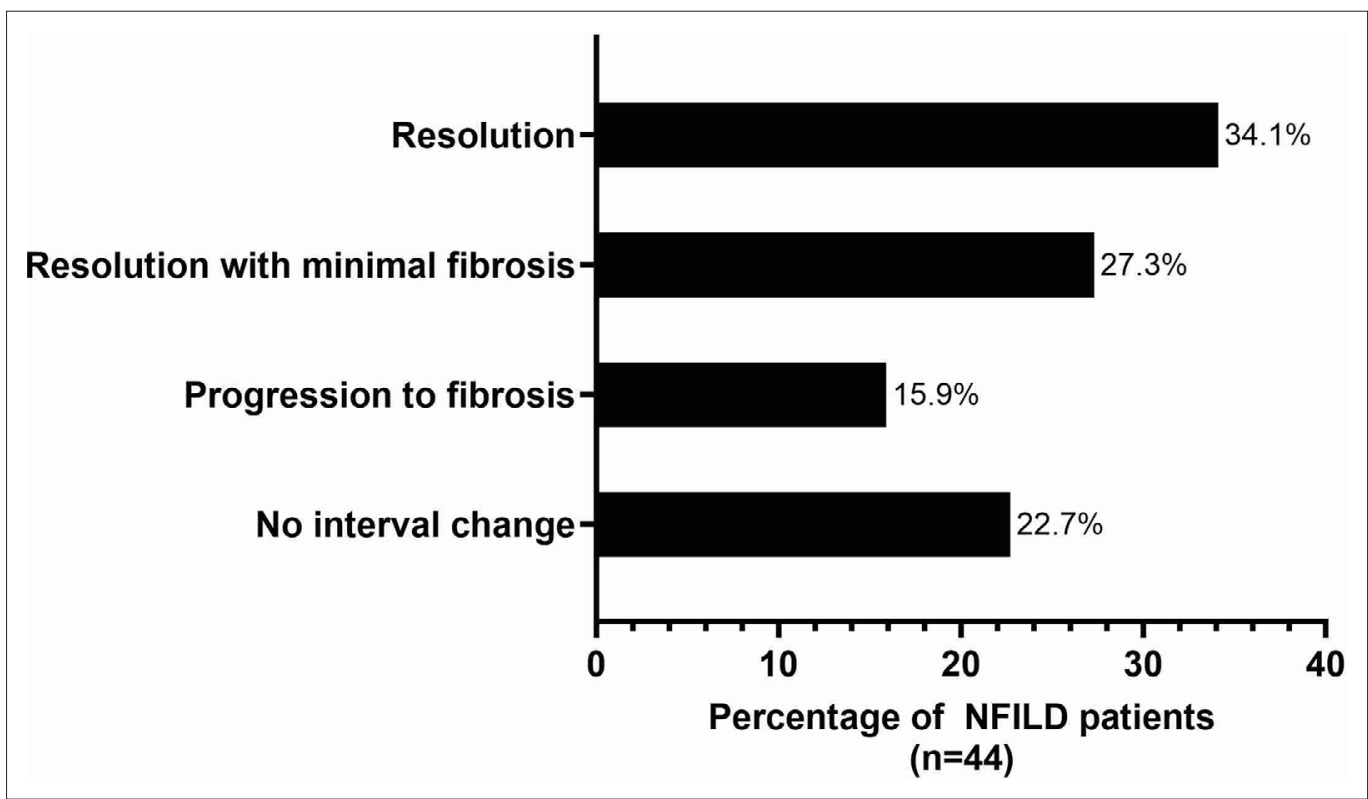

Figure 4 Interval radiological outcomes of patients with NFILD followed-up in the BILD clinic

The median follow-up period by the BILD service following NF cessation was 11.0 months (IQR 6.0-23.0). From baseline to final clinic visit, spirometry showed statistically significant improvements in FVC $(P=0.041)$, TLCO $(P=0.005)$, and KCO $(P=0.006)$ (Figure 3$)$.

There was no significant change in resting oxygen saturation (mean $95.4 \pm 2.1 \%$ versus $96.1 \pm 1.7 \%, P$ =0.68), 6MWT (median [IQR] absolute distances $280.0 \mathrm{~m}$ [211.3-345.5] versus $280.0 \mathrm{~m}$ [220.0-320.0], $P=0.14$ ), or desaturation during 6MWT (median [IQR] 90.0\% [86.8-93.0] versus 92.5\% [91.0-93.0], $P=$ 0.34 ) between initial visit and final visits. Notably, there was significant improvement in MRC dyspnoea score (mean $3.3 \pm 1.2$ versus $2.9 \pm 1.3, P=0.011$ ).

The patterns of fibrosis at baseline HRCT included cellular non-specific interstitial pneumonia (NSIP) $(13.0 \%, n=6)$, fibrotic NSIP $(17.4 \%, n=8)$, organising pneumonia (OP) $(10.9 \%, n=5)$, fibrotic OP $(2.2 \%, n=1)$, and hypersensitivity pneumonitis $(13.0 \%, n=6)$, with an overlap of features in the remaining 20 patients.

Follow-up HRCT were available in 44 patients, at a median interval of 10.5 months (IQR 5.0-22.0). Interval radiological changes (Figure 4) in most cases showed complete resolution or minimal fibrosis $(61.4 \%, n=27)$. Some showed progression of fibrosis over the follow-up period $(15.9 \%, n=7)$. The remainder of patients showed no change $(22.7 \%, n=10)$.

HRCT radiological outcomes were not statistically significant between patients who were corticosteroid-treated and those who had not received corticosteroids ( $n=29$ and $n=15$, respectively, $P=0.83)$, although the cohorts were small.

Of the 46 patients, four consequently required LTOT and five required AOT.

\section{Discussion}

\section{Summary}

This study has found low awareness of complications and suboptimal monitoring associated with long-term NF treatment, evident from both prescriber self-reporting and patient records. The authors advocate augmentation of current guidelines with a model monitoring plan. The pulmonary outcomes of patients with NFILD serve as a warning of some potential consequences of low awareness and monitoring, even before exploring hepatic impacts.

Inadequate monitoring delays drug cessation and increases toxicity. ${ }^{6}$ The questionnaire revealed low rates of active baseline testing, required to recognise any abnormalities in future monitoring results. However, in practice, many patients may have received 'passive' baseline testing associated with routine health checks. By the authors' definition of baseline monitoring, it has been shown that 
monitoring in practice may be lower than self-reported monitoring. Follow-up monitoring is also low; large proportions of prescribers reported that they 'never' monitor liver or lung complications, and only a minority 'always' monitor these.

The questionnaire also uncovered a discrepancy in perceived responsibilities between primary and secondary care professionals. Some primary care responders expect secondary professionals to perform baseline tests. Furthermore, almost all GPs, compared with approximately half of urologists, considered monitoring to be the prescriber's responsibility. Effective communication between primary and secondary care is vital to ensure high-quality health care. Breakdown of this communication can result in reduced quality of care. ${ }^{13}$

\section{Strengths and limitations}

This study engaged all parties involved in prescribing and monitoring of NF. The questionnaire had an adequate response rate from a large sample population, but remains susceptible to response bias. Despite this, among responders, the results are concerning and suggest a general low awareness and/ or perceived significance of the problem. All LFTs in data collection were considered as undertaken for monitoring purposes. It is not possible to attribute this with certainty; many LFTs are likely to be coincidental given the cohort's advanced age and comorbidities. Hence, deliberate hepatic monitoring was likely overestimated. It should also be acknowledged that GPs often rely on existing clinical information and/or results in decision making about baseline safety for prescribing.

It is recognised that the sample of patients with NFILD was relatively small. Nonetheless, the primary focus of this study was monitoring, not sequelae of complications. There are further limitations with retrospective study that include missing data. The COVID-19 pandemic may have affected the final 4 months of the investigation window, possibly impacting follow-up, treatment routines, or respiratory outcomes for some patients.

\section{Comparison with existing literature}

Although complications of NF have long been recognised, ${ }^{14,15}$ there is a dearth of studies on awareness and/or monitoring for NF complications. Audits examining other medications with known potential pulmonary side effects show a similar monitoring shortfall. ${ }^{16-19}$

Other large NF-related studies have shown similar pulmonary complications. ${ }^{7,15,20-22}$ Demographics of these larger cohorts also matched this cohort in age distribution and sex ratio. ${ }^{4,5,23}$

A small proportion of patients with NFILD demonstrated progressive fibrosis despite cessation. Current literature has raised concerns about irreversible pulmonary sequelae of NF use, ${ }^{8,21,23-25}$ although concurrent ILD diagnosis unrelated to NF cannot be excluded. Most of the cohort were exposed to NF for $>6$ months before complications. Comparing radiological outcomes and duration of exposure presented evidence of a positive correlation between duration and chronic impacts, consistent with Holmberg and colleagues' findings; $47 \%$ of their cohort with chronic respiratory disease developed this following at least 12 months of NF. ${ }^{3}$

\section{Implications for research and practice}

Progressive ILD is a significant outcome from long-term NF administration, as well as being a medicolegal prescribing risk. This warrants improvements in baseline assessment and monitoring of patients prescribed NF. Development of education and explicit guidelines on the risks of NF complications could address the low prescriber awareness highlighted in this study and improve compliance. GPs are urged when prescribing NF to be wary of its hepatic and pulmonary risks.

Most questionnaire responders used CCG and NICE guidelines for monitoring and rated current guidelines poorly (2.2/5). BNF guidelines state that prescribers should 'monitor liver function and monitor for pulmonary symptoms ${ }^{110}$ but there is no reference to baseline testing, nor a suggested monitoring plan. Such guidelines should be augmented, emphasising risks, and detailing monitoring plans.

Novel guidance may include but is not limited to:

1. Clear verbal and written information for patients defining potential side-effects of NF, documenting this provision;

2. Need for baseline oxygen saturations, MRC dyspnoea score, respiratory examination, ideally CXR in last 6 months, and LFT and creatinine clearance in last 12 months; 
3. Need for regular follow-up monitoring: respiratory symptoms, oxygen saturations, MRC dyspnoea score, respiratory examination and LFT, ideally at 3-monthly periods during dosing;

4. Rapid cessation if toxicity is suspected;

5. Rapid accessibility to local hepatology and respiratory services; and

6. Review of automated warning and/or monitoring signals on electronic prescribing platforms.

Spirometry has been deliberately excluded given the challenge of delivering this in the current pandemic. This guidance may be sufficient to detect medication-related toxicity. The authors recognise that this guidance may represent an increased burden on GPs. Before any guidance is finalised, a cost-effectiveness analysis could be undertaken, potentially including a modelling study to ascertain the optimal set of monitoring items, hence determining the most effective way to mitigate risk while recognising feasibility.

A further safeguard is improvement of communication pathways between GPs and urologists, so that all parties are aware that the prescriber is responsible for monitoring. Novel guidance could clarify such recommended communication.

The majority of patients registered at BILD were exposed to NF for $>6$ months before complications. There is need for further research into causative durations, but limiting use to 6 months and/or cycling it with a different antibiotic may prove protective. ${ }^{26}$ Beyond 6 months, clinicians should reconsider the prescription in light of its risks.

\section{Funding}

No funding was received for this study.

Ethical approval

This study was approved locally as a Clinical Effectiveness Project (project application number: CE65008).

Provenance

Freely submitted; externally peer reviewed.

\section{References}

1. Price JR, Guran LA, Gregory WT, McDonagh MS. Nitrofurantoin vs other prophylactic agents in reducing recurrent urinary tract infections in adult women: a systematic review and meta-analysis. Am J Obstet Gynecol 2016; 215(5): 548-560. DOI: https://doi.org/10.1016/j.ajog.2016.07.040

2. Bristol, North Somerset and South Gloucestershire Clinical Commissioning Group. Recurrent UTIs in women; https://remedy.bnssgccg.nhs.uk/media/4930/recurrent-utis-in-women-version-21.pdf (accessed 22 Sep 2021).

3. Holmberg L, Boman G, Böttiger LE, et al. Adverse reactions to nitrofurantoin. Analysis of 921 reports. Am J Med 1980; 69(5): 733-738. DOI: https://doi.org/10.1016/0002-9343(80)90443-x

4. Holmberg L, Boman G. Pulmonary reactions to nitrofurantoin. 447 cases reported to the Swedish Adverse Drug Reaction Committee 1966-1976. Eur J Respir Dis 1981; 62(3): 180-189.

5. Claussen $\mathrm{K}$, Stocks $\mathrm{E}$, Bhat $\mathrm{D}$, et al. How common are pulmonary and hepatic adverse effects in older adults prescribed nitrofurantoin? J Am Geriatr Soc 2017; 65(6): 1316-1320. DOI: https://doi.org/10.1111/jgs.14796

6. Muller $A E$, Verhaegh $E M$, Harbarth $S$, et al. Nitrofurantoin's efficacy and safety as prophylaxis for urinary tract infections: a systematic review of the literature and meta-analysis of controlled trials. Clin Microbiol Infect 2017; 23(6): 355-362. DOI: https://doi.org/10.1016/j.cmi.2016.08.003

7. Kabbara WK, Kordahi MC. Nitrofurantoin-induced pulmonary toxicity: a case report and review of the literature. $J$ Infect Public Health 2015; 8(4): 309-313. DOI: https://doi.org/10.1016/j.jiph.2015.01.007

8. Singh J, Dinkar A, Atam V, et al. Drug reaction with eosinophilia and systemic symptoms syndrome associated with nitrofurantoin. J Res Pharm Pract 2016; 5(1): 70-73. DOI: https://doi.org/10.4103/2279-042X.176560

9. Kapral N, Saxena R, Sule AA, Markle B. Nitrofurantoin: friend or foe? BMJ Case Rep 2018; 2018: bcr2018225629. DOI: https://doi.org/10.1136/bcr-2018-225629

10. Joint Formulary Committee. British National Formulary. Nitrofurantoin: monitoring requirements; https://bnf.nice. org.uk/drug/nitrofurantoin.html\#monitoringRequirements (accessed 22 Sep 2021).

11. National Institute for Health and Care Excellence. Urinary tract infection (recurrent): antimicrobial prescribing. NG112; https://www.nice.org.uk/guidance/ng112 (accessed 17 Sep 2021).

12. Medica Protection. Complications of nitrofurantoin. 2018; https://www.medicalprotection.org/uk/articles/ complications-of-nitrofurantoin (accessed 17 Sep 2021).

13. Sutcliffe KM, Lewton E, Rosenthal MM. Communication failures: an insidious contributor to medical mishaps. Acad Med 2004; 79(2): 186-194. DOI: https://doi.org/10.1097/00001888-200402000-00019 
14. Fisk AA. Brief recording: anaphylactoid reaction to nitrofurantoin. N Engl J Med 1957; 256(22): 1054. DOI: https:// doi.org/10.1056/NEJM195705302562207

15. Israel KS, Brashear RE, Sharma HM, et al. Pulmonary fibrosis and nitrofurantoin. Am Rev Respir Dis 1973; 108(2): 353-356. DOI: https://doi.org/10.1164/arrd.1973.108.2.353

16. Goldschlager N, Epstein AE, Naccarelli GV, et al. A practical guide for clinicians who treat patients with amiodarone: 2007. Heart Rhythm 2007; 4(9): 1250-1259. DOI: https://doi.org/10.1016/j.hrthm.2007.07.020

17. Jackevicius CA, Tom A, Essebag V, et al. Population-level incidence and risk factors for pulmonary toxicity associated with amiodarone. Am J Cardiol 2011; 108(5): 705-710. DOI: https://doi.org/10.1016/j.amjcard.2011.04. 024

18. Lavon O, Goldman R. Adherence to monitoring guidelines of amiodarone adverse reactions. Health Serv Res Manag Epidemiol 2019; 6: 2333392819844635 DOI: https://doi.org/10.1177/2333392819844635

19. Burgess C, Blaikie A, Ingham T, et al. Monitoring the use of amiodarone: compliance with guidelines. Intern Med J 2006; 36(5): 289-293. DOI: https://doi.org/10.1111/j.1445-5994.2006.01068.x

20. Mir E, Malik JA, Lone SA, et al. Spontaneous resolution of nitrofurantoin-induced chronic pulmonary toxicity presenting with respiratory failure. Adv Respir Med 2017; 85(6): 333-338. DOI: https://doi.org/10.5603/ARM.2017. 0057

21. Sakata KK, Larsen BT, Boland JM, et al. Nitrofurantoin-induced granulomatous interstitial pneumonia. Int J Surg Pathol 2014; 22(4): 352-357. DOI: https://doi.org/10.1177/1066896913492849

22. Almeida P, Seixas E, Pinheiro B, et al. Consider nitrofurantoin as a cause of lung injury. Eur J Case Rep Intern Med 2019; 6(11): 001295. DOI: https://doi.org/10.12890/2019_001295

23. Mendez JL, Nadrous HF, Hartman TE, Ryu JH. Chronic nitrofurantoin-induced lung disease. Mayo Clin Proc 2005; 80(10): 1298-1302. DOI: https://doi.org/10.4065/80.10.1298

24. Madani $Y$, Mann B. Nitrofurantoin-induced lung disease and prophylaxis of urinary tract infections. Prim Care Respir J 2012; 21(3): 337-341. DOI: https://doi.org/10.4104/pcrj.2012.00059

25. Willcox PA, Maze SS, Sandler M, et al. Pulmonary fibrosis following long-term nitrofurantoin therapy. South Afr Med J 1982; 61(19): 714-717.

26. Lin DC-yen, Bhally H. Nitrofurantoin-induced interstitial lung disease. N Z Med J 2007; 120(1263): U2753. 